Stimmrechten des zu finanzierenden MVZ-Rechtsträgers verschaffen, oder zu unzulässiger Beteiligung von Kapitalgebern am Gewinn des MVZ-Rechtsträgers führen.

Nicht zu unterschätzen sind auch die richterrechtlich geprägten Institute der Durchgriffshaftung von faktischen Gesellschaftern von Kapitalgesellschaften und die erklärte Absicht des Gesetzgebers, den angeblichen ,schädlichen“"Einfluss von Fremdkapitalgeber auf MVZ zurückdrängen zu wollen. Stellen Fremdkapitalgeber einem MVZ-Rechtsträger auf ei- gene Rechnung das Grund- oder das Stammkapital zur Verfügung und treten sie im Außenverhältnis so auf, dass Gesellschafter eines MVZ-Rechtsträgers lediglich als Strohmänner oder Strohfrauen einzuordnen sind, können die bezeichneten Fremdkapitalgeber als faktische Gesellschafter des MVZRechtsträgers nach den Grundsätzen der Durchgriffshaftung in Anspruch genommen werden. Ferner kommt die Haftung von bezeichneten Fremdkapitalgebern als faktische Geschäftsführer des betroffenen MVZ-Rechtsträgers in Betracht.

\title{
AU-Schein nach Fernuntersuchung
}

\author{
zugleich Anmerkung zu LG Hamburg, Urt. v. 3.9.2019 - 406 HKO 56/19 (in diesem Heft, S. 391)
}

\section{Erik Hahn}

\section{Das Modell „AU-Schein nach Fernuntersuchung“}

Das Urteil des LG Hamburg befasst sich mit einem wettbewerbsrechtlichen Unterlassungsbegehren nach $₫ 8$ Abs. 1 UWG gegen den Betreiber einer Internetplattform in der Rechtsform einer $\mathrm{GmbH}$, der die Ausstellung von AUBescheinigungen ohne physischen Arzt-Patienten-Kontakt anbietet. Der in den FAQs als „Besteller“" (nomen est omen?) bezeichnete „Patient“" wird auf der Internetseite durch ein Auswahlmenü geführt und muss dort zunächst Angaben zu der von ihm selbst vermuteten Krankheit, etwaigen Symptomen und dem Beginn der Beschwerden tätigen ${ }^{2}$. Außerdem kann er in einem vorgegebenen Rahmen selbst auswählen, für wie viele Tage er sich arbeitsunfähig fühlt und ob er eine rückwirkende Bescheinigung benötigt. Nach der Bestätigung der Risiko- und Ausschlusshinweise, der Eingabe persönlicher Daten und der Auswahl der Zahlungsmethode erhält der Besteller einen SMS-Code, der den Zugang zur persönlichen AU-Bescheinigung im pdf-Format gewährt. Die im ursprünglichen Konzept noch vorgesehene und auch im Tatbestand des Urteils angesprochene Übermittlung der Bescheinigung über den Messenger-Dienst „WhatsApp“ wird ausweislich der - inzwischen wohl geänderten ${ }^{3} \mathrm{FAQs}$ nicht mehr angeboten.

\section{Der Verstoß gegen $₫ 25$ S. $1 \mathrm{HmbBOÄ}$ als Anknüpfung für die $\mathbb{S} 3$ und 3a UWG}

Die im Wettbewerbsrecht angesiedelte Entscheidung des LG Hamburg findet den Schwerpunkt ihrer juristischen Auseinandersetzung über die $\$ \$ 3$ und 3 a UWG im ärztlichen Berufsrecht. Unlautere geschäftliche Handlungen sind nach $\$ 3$ Abs. 1 UWG unzulässig. Darunter fallen nach $\$ 3$ Abs. 2 UWG zunächst solche geschäftlichen Handlungen, die sich an Verbraucher richten oder diese erreichen, wenn sie nicht der unternehmerischen Sorgfalt entsprechen und dazu geeignet sind, das wirtschaftliche Verhalten des Verbrauchers wesentlich zu beeinflussen. Konkretisierend ${ }^{4}$ knüpft \3a UWG die Unlauterkeit an einen Verstoß gegen gesetzlich verankerte Marktverhaltensregeln, wenn dieser geeignet ist, die Interessen von Verbrauchern, sonstigen

\section{Prof. Dr. iur. Erik Hahn,}

Professur für Zivilrecht, Medizinrecht, Wirtschafts-

und Immobilienrecht

sowie Mitglied des Instituts für Gesundheit, Altern und Technik, Hochschule Zittau/Görlitz,

Theodor-Körner-Allee 16, 02763 Zittau, Deutschland
Marktteilnehmern oder Mitbewerbern spürbar zu beeinträchtigen. Beide Varianten sieht das LG Hamburg unter Verweis auf die Berufsordnung der Hamburger Ärzte als erfüllt an, obwohl nichtärztliche Leistungsanbieter nicht ${ }^{5}$ zu deren Adressatenkreis zählen. Das unlautere Verhalten erkennt das Gericht indes in der Bewerbung und Organisation des Geschäftsmodells, das geeignet sei, Patienten vom Praxisbesuch abzuhalten und damit das Verbraucherverhalten zu lenken. Der Portalbetreiber wäre somit zumindest hinsichtlich des Rechtsbruchs nach $₫ 3$ a UWG als Teilnehmer einzustufen, dessen Tätigkeit durch den ärztlichen Berufsrechtsversto $ß$ „infiziert" wird. Dabei wäre allerdings vor dem Hintergrund der momentan erheblich umstrittenen $^{6}$ rechtlichen Bewertung von Fernattesten eine Auseinandersetzung mit dem Teilnehmervorsatz erforderlich gewesen. Dieser muss sich auch auf die Umstände beziehen, die die Rechtswidrigkeit der Haupttat begründenden? ${ }^{7}$. Erörterungen dazu finden sich im Urteil nicht.

Die Prüfung eines berufsrechtlichen Rechtsverstoßes i. S.v. $\$ 3$ a UWG (vormals $\$ 4$ Nr. 11) überzeugt aber zumindest insoweit, als es sich bei einzelnen (nicht allen) Vorgaben der LBOs (nicht der MBO-Ä) ${ }^{8}$ um Marktverhaltensregeln handeln kann?. Für das vom materiellen Gesetzesbegriff erfasste Satzungsrecht gilt das jedenfalls dann, wenn die erforderliche Qualität einer „,gesetzlichen Vor-

1) Vgl. https://www.au-schein.de/faq, abgerufen am 6.2.2020.

2) Vgl. https://au-app.au-schein.de, abgerufen am 6.2.2020.

3) Im FAQ-Register lautet der Menüpunkt (noch?): „Kann ich den Service auch ohne WhatsApp nutzen?". Er ist verlinkt auf den Unterpunkt: „Kann ich den Service mit WhatsApp nutzen?“ mit der Antwort: ,Nein! Die Kommunikation zwischen Ihnen als Patient und dem Arzt erfolgt immer ganz ohne WhatsApp." (https:// www.au-schein.de/faq, abgerufen am 6.2.2020).

4) Schaffert, in: Heermann/Schlingloff (Hrsg.), MüKo/UWG, 3. Aufl. 2020, \$3a, Rdnr. 37.

5) So etwa für Krankenhäuser: OLG Düsseldorf, Urt. v. 16. 11.2004 -20 U 30/04.

6) Vgl. dazu Braun, GesR 2018, 409, 412; Hahn, ZMGR 2018, 279 ff.; Heider, NZA 2019, 288 ff.; Möller/Flöter, ArbRAktuell 2019, 501 ff.

7) Fritzsche, in: Heermann/Schlingloff (Hrsg.), MüKo/UWG, 2. Aufl. 2014, \8, Rdnr. 243; OLG Düsseldorf, MedR 2005, 169.

8) Köhler, in: Köhler/Bornkamm/Feddersen (Hrsg.), UWG, 38. Aufl. 2020, \&3a, Rdnr. 1.132.

9) Vgl. Fritzsche, in: Spickhoff(Hrsg.), Medizinrecht, 3. Aufl. 2018, §3a UWG, Rdnr. 2; Schaffert, in: Heermann/Schlingloff (Hrsg.), MüKo/ UWG, 3. Aufl. 2020, \$3a, Rdnr. 173; v. Jagow, in: Harte-Bavendamm Henning-Bodewig (Hrsg.), UWG, 4. Aufl. 2016, §3a, Rdnr. 55; BGH, GRUR 2015, 1237, 1238f.; OLG München, Urt. v. 8. 10.2015-6 U 1509/15 -; LG München, GRUR-RR 2012, 260, 261. 
schrift" über die Kompetenzgrundlage der jeweils einschlägigen Heilberufsgesetze vermittelt wird ${ }^{10}$. In Hamburg ist dieses im speziellen Fall $\$ 28$ Abs. 2 Nr. 2 HmbKGH (Regelungen [...] zu [...] der Ausstellung von Gutachten und Zeugnissen). Auch die Vorgaben der Landesärztekammern zur Fernbehandlung sind Marktverhaltensregeln i. S. v. \$3a $\mathrm{UWG}^{11}$.

In den Entscheidungsgründen umschifft das Gericht den im Tatbestand zumindest angesprochenen $\$ 9 \mathrm{HWG}$ und die damit zwangsläufig verbundene heikle Diskussion um die berufsrechtlichen Grenzen ${ }^{12}$ der ärztlichen Fernbehandlung sowie eine gegebenenfalls erforderliche Reduktion ${ }^{13}$ des Werbeverbots. Seine Auffassung stützt es vielmehr auf die in $\$ 25 \mathrm{~S}$. $1 \mathrm{HmbBOÄ}$ verankerte Pflicht, bei der Ausstellung ärztlicher Gutachten und Zeugnisse mit der notwendigen Sorgfalt zu verfahren und nach bestem Wissen die eigene ärztliche Überzeugung auszusprechen. Dieses sei nur zu gewährleisten, wenn Arzt und Patient in der Praxis oder bei einem Hausbesuch aufeinandertreffen. Ohne diese notwendige Grundbedingung sei es dem Arzt dagegen unmöglich, die bestehende oder behauptete Erkrankung sicher festzustellen. Dieser Makel sei auch nicht durch die Möglichkeit von Rücksprachen per Telefon oder VideoChat zu beheben. Argumentativ war für das Gericht in besonderem Maße ausschlaggebend, dass die Krankschreibung die Grundlage für die Entgeltfortzahlungspflicht des Arbeitgebers nach $\$ 7$ Abs. 1 Nr. 1 und $\$ 5$ Abs. 1 EntgFG bildet, bei der auch die Schwere der Erkrankung sicher ermittelt werden müsse.

\section{Die vollständige Verdrängung von} \7 Abs. 3 S. 3 HmbBOÄ durch $\ 25$ S. 1 HmbBOÄ?

Vor dem Hintergrund des oben beschriebenen Geschäftsmodells überzeugt - zumindest bei unterstelltem Vorliegen aller subjektiven Voraussetzungen (s. o.) - nur das Ergebnis der Entscheidung. Die apodiktische Begründung wirkt dagegen zu undifferenziert. $\$ 25 \mathrm{~S}$. $1 \mathrm{HmbBOÄ}$ verlangt die Wahrung der notwendigen Sorgfalt bei der Ausstellung ärztlicher Zeugnisse. Der Inhalt dieser Sorgfaltspflichtanforderung ist unbestimmt und muss im Zusammenhang mit anderen medizinrechtlichen Regelungen - allen voran solchen des Berufsrechts - ermittelt werden. Dabei fällt zunächst auf, dass das LG Hamburg den 2018 grundlegend reformierten $\$ 7$ Abs. 4 S. 3 MBO-A (ausschließliche Fernbehandlung) - der in Hamburg zum 1.9.2019 (Beschluss v. 8.4.2019) wortgleich in $\$ 7$ Abs. 3 S. 3 HmbBOÄ umgesetzt wurde - ebenfalls nur im Tatbestand erwähnt, in den Entscheidungsgründen aber völlig hinter $\$ 25$ S. 1 HmbBOÄ zurücktreten lässt. Das könnte selbst mit dem Argument einer Einstufung als Spezialvorschrift ${ }^{14}$ für ärztliche Zeugnisse nicht überzeugen. Zum einen ist das streitgegenständliche Geschäftsmodell (AU-Bescheinigung ohne Praxisbesuch) gerade im Bereich der ausschließlichen Fernbehandlung angesiedelt. Zum anderen formuliert das LG Hamburg in seiner Entscheidung keine allgemeinen Erwägungen zur ärztlichen Sorgfaltspflicht bei der Ausstellung von AU-Bescheinigungen, sondern versteigt sich in konkrete Ausführungen zur ärztlichen Vertretbarkeit von Fernattesten. Dabei verweist es darauf, dass es auch bei herkömmlichen Arbeitsunfähigkeitsuntersuchungen in der Praxis immer wieder zu Fällen kommen könne, bei denen die Bescheinigung ohne hinreichende Untersuchung ausgestellt wird. Auch in diesen abstrakt thematisierten Fällen sieht das LG Hamburg sorgfaltswidriges Verhalten im Sinne von $\$ 25$ S. $1 \mathrm{HmbBOA}$ als gegeben an. Das zur Beurteilung stehende Geschäftsmodell lehnt das Gericht indes explizit mit dem Argument der Insuffizienz ausschließlich fernkommunikativer Untersuchungsformen ab. Für diese Frage ist aber $₫ 7$ Abs. 3 S. 3 HmbBOÄ - bei einem engen Verständnis des Behandlungsbegriffs ( $\neq$ Arbeitsunfä- higkeitsuntersuchung) - zumindest ergänzend, wenn nicht sogar - bei einem weiten Verständnis (Untersuchung als Teil der „Behandlung“) - als lex specialis heranzuziehen. Nach dieser Vorschrift ist eine ausschließliche Beratung oder Behandlung über Kommunikationsmedien im Einzelfall erlaubt, wenn dies ärztlich vertretbar ist und die erforderliche ärztliche Sorgfalt gewahrt wird. Parallel zur berufsrechtlichen Entwicklung hat auch der Gesetzgeber etwa durch die Streichung ${ }^{15}$ von $\$ 48$ Abs. 1 S. 2 AMG a.F. (Verbot der Arzneimittelabgabe nach Fernverschreibung) - der ausschließlichen Fernbehandlung den Weg bereitet. Im Vertragsarztrecht wurde die Videosprechstunde schon seit 2017 vorübergehend mit eigenen GOPs ausgestattet und inzwischen zum 1.10.2019 in das allgemeine System der Versichertenpauschalen überführt ${ }^{16}$. Außerdem wurde inzwischen auch das im Urteil ebenfalls genannte Verbot der Werbung für eine ausschließliche Fernbehandlung nach \$9 HWG - ebenfalls eine Marktverhaltensregel i.S.v. \$3a UWG $-{ }^{17}$ durch das $D_{V G}{ }^{18}$ zum 19.12.2019 dahingehend entschärft, dass berufsrechtlich zulässige Fernbehandlungsformen davon ausgenommen sind. Bekannt war dieses Änderungsvorhaben bereits seit der Veröffentlichung des entsprechenden Referentenentwurfs im Mai $2019^{19}$.

Die ausschließliche Fernbehandlung ist - und war bereits im Zeitpunkt der Entscheidungsfindung - in der ärztlichen Versorgung angekommen. Unter Beachtung der Vertretbarkeit im Einzelfall ist es Ärzten gestattet, gesundheitsbezogene Entscheidungen auf der Basis von Ferndiagnosen mit dem Patienten über Fernkommunikationsmittel zu erörtern, diesen per Fernverschreibung mit Arzneimitteln zu versorgen und Behandlungsempfehlungen auszusprechen ${ }^{20}$. Weshalb all dieses möglich sein soll, ohne daraus per se einen Sorgfaltsverstoß herzuleiten, während der ausschließliche Fernkontakt für die Arbeitsunfähigkeitsfeststellung generell zu unsicher sei, erschließt sich weder aus der Begründung des LG Hamburg noch aus der Gesamtschau der \$S 7 Abs. 3 und 25 S. 1 HmbBOÄ.

\section{IV. Änderung der Rechtslage zwischen Verhandlung und Urteil}

Dem LG Hamburg ist zugutezuhalten, dass es mit seinem Urteil in eine die Sache zusätzlich verkomplizierende Übergangsphase geraten ist. Am 20.8.2019, dem Tag der letzten mündlichen Verhandlung und maßgeblichen ${ }^{21}$ Zeit-

10) Zur wettbewerbsrechtlichen Bedeutung standesrechtlicher Regelungen ohne gesetzliche Grundlage vgl. BGH, NJW-RR 1989, $1385 \mathrm{f}$.

11) OLG München, Urt. v. 8.10.2015-6 U 1509/15 -, Rdnrn. 37 ff.

12) Vgl. dazu exemplarisch Dierks, MedR 2016, 405 ff.; Gruner, GesR 2017, 288 ff.; Hahn, Telemedizin - Das Recht der Fernbehandlung, 2019; ders., MedR 2018, $384 \mathrm{ff}$.; Kalb, GesR 2018, 481 ff.; Katzenmeier, MedR 2019, 259 ff.; ders. NJW 2019, 1769 ff.; Spickhoff, MedR 2018, 535 ff.; Stellpflug, GesR 2019, 76ff.; Wolf, GuP 2018, $129 \mathrm{ff}$.

13) Braun, MedR 2018, 563, 566; Hahn, MedR 2018, 384, 389; a. A Kuhn/Heinz, GesR 2018, 691, 693.

14) So wohl zu verstehen bei Scholz, in: Spickhoff (Hrsg.), Medizinrecht, 3. Aufl. 2018, §7 MBO-Ä, Rdnr. 17:, §25 S. 1 bleibt allerdings unberührt".

15) BGBl. I 2019, S. 1202, 1203

16) Bewertungsausschuss-Ärzte, DÄBl. 2019, A-2038ff.

17) Schaffert, in: Heermann/Schlingloff (Hrsg.), MüKo/UWG, 3. Aufl. 2020, \3a, Rdnr. 342; v. Jagow, in: Harte-Bavendamm/HenningBodewig (Hrsg.), UWG, 4. Aufl. 2016, Einleitung I, Rdnr. 60.

18) BGB1. I S. 2562, 2583

19) DVG-RefE v. 15.5.2019, S. 31, https://tinyurl.com/yyrqbevr, abgerufen am 6.2.2020.

20) Zur Zulässigkeit der Fernaufklärung vgl. Hahn, MedR 2020 , 16 ff.; Katzenmeier, NJW 2019, 1769, 1773; Kaeding, MedR 2019 , 288, 289; Stellpflug, GesR 2019, 76, 78.

21) Fritzsche, in: Heermann/Schlingloff (Hrsg.), MüKo/UWG, 2. Aufl. 2014, \$8, Rdnr. 38; BGH, GRUR 2010, 455, 456. 
punkt für die bei einem Unterlassungsbegehren nach $₫ 8$ Abs. 1 UWG erforderliche Wiederholungsgefahr, war zwar $\$ 7$ Abs. 4 S. $3 \mathrm{MBO}-\ddot{\mathrm{A}}$ schon ein Jahr reformiert. Dieser ist aber nur eine Modellnorm und damit nicht verbindlich ${ }^{22}$. Der maßgebliche $\$ 7$ Abs. 3 S. 3 HmbBOÄ trat dagegen erst zwölf Tage später, am 1.9.2019, jedoch noch zwei Tage vor dem Urteil, am 3.9.2019, in Kraft. Die Grundlagen der Entscheidung fallen damit noch in den Zeitraum der alten Rechtslage. Andererseits wurde der bereits am 8.4.2019 gefasste Beschluss der Hamburger Delegiertenversammlung zur Änderung von $\$ 7$ HmbBOÄ schon am 20.6.2019 durch die zuständige Aufsichtsbehörde genehmigt und zudem bereits im August - und damit zum Zeitpunkt der Verhandlung - im Hamburger Ärzteblatt veröffentlicht ${ }^{23}$. Die Änderung der Rechtslage war damit also zum Zeitpunkt der Verhandlung schon sicher angekündigt und hätte daher bei der Beurteilung der Wiederholungsgefahr berücksichtigt ${ }^{24}$ werden müssen. Das LG Hamburg scheint die spezielle Fernbehandlungsregelung jedoch sowohl in alter als auch in neuer Fassung überhaupt nicht für entscheidungserheblich gehalten zu haben und konnte dieser Frage daher - zumindest konsequent - ,ausweichen“.

\section{V. Ärztliche Vertretbarkeit von Ferndiagnosen auch bei AU-Bescheinigungen?}

\section{Die grundsätzliche Insuffizienz von Ferndiagnosen}

Neben der ausschließlichen Anknüpfung der berufsrechtlichen Fragestellung an $\$ 25$ S. 1 HmbBOÄ bietet auch die medizinspezifische Sachverhaltsaufklärung des LG Hamburg Anlass zu Zweifeln. Das gilt insbesondere für die scheinbar allgemeingültige Aussage, ein Arzt könne sich nur bei einem Praxiskontakt oder Hausbesuch einen unmittelbaren Eindruck von dem Gesundheitszustand des $\mathrm{Pa}-$ tienten verschaffen. Diese Annahme ist zum einen schon nicht mit der reformierten Grundaussage von $\$ 7$ Abs. 4 S. $3 \mathrm{MBO}-\ddot{\mathrm{A}}$ (ärztliche Vertretbarkeit im Einzelfall) zu vereinbaren. Zum anderen reißt sie eigentlich überwundene Diskussionsgräben zur grundsätzlichen Zulässigkeit ausschließlicher Fernbehandlungen erneut auf: So hat sich etwa die in dieser Sache ohnehin restriktiv ausgerichtete Landesärztekammer Brandenburg in einer Pressemitteilung dahingehend geäußert, dass sie sich durch das - eigentlich nur AU-Bescheinigungen betreffende - Urteil in ihrer ablehnenden Grundhaltung gegenüber ausschließlichen Fernbehandlungen bestätigt sehe. Das Gericht habe „festgestellt, dass eine Diagnosestellung aus der Ferne ohne unmittelbaren Arzt-Patienten-Kontakt grundsätzlich gegen die rechtlich gebotene ärztliche Sorgfalt verstößt." 25 Das war jedoch weder Gegenstand der vom LG Hamburg zu entscheidenden Frage, noch entspräche diese Ansicht der Rechtslage nach $\$ 7$ Abs. 3 S. 3 HmbBOÄ. Die durch die Bundesärztekammer repräsentierte Ärzteschaft vertritt zudem auch zur konkreten Problematik der Ausstellung von AU-Bescheinigungen eine wesentlich differenziertere Position $^{26}$. In ihrem vom 22.3.2019 stammenden Hinweispapier zur Fernbehandlung findet sich etwa die Aussage, dass es bei $\int 25 \mathrm{~S} .1 \mathrm{MBO}-\ddot{\mathrm{A}}$ darauf ankomme, dass der Arzt seine Überzeugung von der krankheitsbedingten Arbeitsunfähigkeit auch ohne die im persönlichen Kontakt zur Verfügung stehenden Untersuchungsmittel erlangen könne ${ }^{27}$. Das ist kein Ausschluss, sondern nur eine Einschränkung, die zudem in $\$ 7$ Abs. 4 S. 3 MBO- ̈̈ (,ärztliche Vertretbarkeit") ohnehin angelegt ist. Umgekehrt ist diesem Statement der Bundesärztekammer aber auch kein generelles Bekenntnis der Ärzteschaft zu Fernattesten zu entnehmen. An derselben Stelle ${ }^{28}$ findet sich schließlich ein Hinweis auf den beschränkten Beweiswert von AU-Bescheinigungen, die ohne vorausgegangene Untersuchung und allein aufgrund der Angaben des Patienten ausgestellt werden.

\section{1976 ist nicht 2020 und das Internet}

ist kein Kommunikationsverzicht

Der letztgenannte Hinweis der Bundesärztekammer auf die eingeschränkte Aussagekraft stützt sich auf ein in diesem Kontext viel zitiertes Urteil ${ }^{29}$ des BAG aus dem Jahr 1976, das jedoch zur vorliegenden Konstellation bei genauer Betrachtung überhaupt keine zwingende Aussage trifft. Zum einen muss berücksichtigt werden, dass sich die Fernkommunikationsmöglichkeiten zwischen Arzt und Patient außerhalb von Praxis- und Hausbesuchen in den letzten 44 Jahren (das World Wide Web stammt erst aus den 90er-Jahren) wesentlich verändert haben. Zum anderen befasst sich die BAG-Entscheidung mit der Ausstellung ärztlicher Atteste ohne (erneute) Untersuchung des Arbeitnehmers ${ }^{30}$. Die Ausstellung von AU-Bescheinigungen bei einer ausschließlichen Fernbehandlung muss jedoch nicht auf Basis eines vollständigen Untersuchungsverzichts erfolgen, sondern kann sich zumindest auf eine - in den zur Verfügung stehenden Mitteln naturgemäß eingeschränkte - Fernuntersuchung stützen. Insbesondere der Einsatz eines Video-Chats kann hier eine rudimentäre optische oder akustische Befunderhebung ermöglichen. $\mathrm{Ob}$ das für den konkreten Patienten und die konkrete Erkrankung ausreichend ist, muss der Arzt im jeweiligen Einzelfall ( $\$ 7$ Abs. 4 S. $3 \mathrm{MBO}-\ddot{\mathrm{A}}$ ) individuell prüfen. Die Fernarbeitsunfähigkeitsuntersuchung ist damit ebenso wenig generell ausgeschlossen, wie sie auch nicht in jedem Einzelfall möglich ist. Gerade der letztgenannten Einschränkung scheint das streitgegenständliche Geschäftsmodell - jedenfalls soweit dieses nach der Beschreibung beurteilt werden kann - nicht genügt zu haben. Zumindest sind keine hinreichenden ${ }^{31}$ Vorkehrungen ersichtlich, die dem Eindruck eines Automatismus zwischen dem Besuch der Website und dem Erhalt der AU-Bescheinigung entgegenwirken können. Das betrifft aber nicht das Modell der „Fernatteste“ als solches, sondern nur die Bewertung des konkreten Geschäftsmodells, das sich (wohl vorrangig?) auf die Auswahl von vorgegebenen Antwortmöglichkeiten durch den AU-Besteller beschränkt.

\section{Sonderrolle der Arbeitsunfähigkeitsuntersuchung?}

An dieser Einschätzung ändert auch der im Urteil des LG Hamburg mehrfach zu findende Hinweis auf die (nur?) bei Arbeitsunfähigkeitsuntersuchungen besonders erforderliche sichere Feststellung nichts. Zum einen dient die in $\$ 7$ Abs. 4 S. 3 MBO-Ä vorausgesetzte ärztliche Vertretbarkeit der ausschließen Fernbehandlung in erster Linie dem Schutz des Patienten ${ }^{32}$. Dagegen sichern die speziellen An-

22) Scholz, in: Spickhoff (Hrsg.), Medizinrecht, 3. Aufl. 2018, Vorbem. zur MBO- $\overparen{A}$, Rdnr. 1.

23) Vgl. Hamburger Ärzteblatt 08/2019, 44.

24) Fritzsche, in: Heermann/Schlingloff(Hrsg.), MüKo/UWG, 2. Aufl. 2014, §8, Rdnr. 73.

25) LÄK Brandenburg, https://tinyurl.com/u8rbp72, abgerufen am 6.2.2020. Mit ähnlichem Fazit: Menke, FD-MedizinR 2020, 424324.

26) BÄK, DÄB1. 2019, A-978, grundsätzlich ablehnend dagegen noch 121. DÄT, Protokoll IV-01, IV-04, S. 1.

27) B̈̈K, DÄBl. 2019, A-978.

28) BÄK, DÄBl. 2019, A-978.

29) BAG, NJW 1977, $350 \mathrm{f}$.

30) BAG, NJW 1977, 350.

31) Zwar werden im „Bestellvorgang“ Ausschlusskriterien abgeprüft, bei deren Vorliegen der Patient auf einen Praxisbesuch verwiesen wird. Für die grundsätzlich erfassten Szenarien scheint aber kein hinreichendes Verifizierungsverfahren für die Bestellerangaben vorgelegen zu haben, vgl. dazu. https://www.au-schein.de/faq, abgerufen am 6.2.2020.

32) OLG München, Urt. v. 8.10.2015 - 6 U 1509/15 -, Rdnr. 48. 
forderungen an die Arbeitsunfähigkeitsuntersuchung das Interesse des Arbeitgebers an belastbaren ${ }^{33}$ Informationen über die Einsatzfähigkeit seines Mitarbeiters und in einem zweiten Schritt - zumindest bei gesetzlich Versicherten die Wirtschaftlichkeit ${ }^{34}$ ( $(12$ Abs. 1 SGB V) der erbrachten Krankengeldleistung nach $\$ 46$ SGB V und damit letztlich die Stabilität der gesetzlichen Krankenversicherung. Ein absolutes Übergewicht des letztgenannten Ziels, dass eine völlig andere Risikobewertung erfordern würde, drängt sich zumindest nicht unmittelbar auf. Zum anderen finden sich weder im EntgFG noch in den korrespondierenden Vorschriften des Vertragsarztrechts ausdrückliche Regelungen, die die Ausstellung einer AU-Bescheinigung nach ausschließlichem Fernkontakt generell verbieten.

Nach der bis zum 8.3.2020 geltenden Fassung von $\$ 31$ BMV-Ä sowie nach $\$ 15$ BMV-Z darf bzw. durfte die AUBescheinigung nur auf Basis einer ärztlichen Untersuchung ausgestellt werden. Das schließt die Fernuntersuchung nicht explizit aus. Außerdem verweisen beide Vorschriften auf die Arbeitsunfähigkeitsrichtlinie des G-BA, die auch allgemein $^{35}$ zur Konkretisierung des berufsrechtlichen Sorgfaltsbegriffs aus $₫ 25 \mathrm{~S}$. $1 \mathrm{MBO}-\ddot{\mathrm{A}}$ herangezogen wird. Diese verlangt in ihrem $\ 4$ Abs. $1 \mathrm{~S} .2$ ebenfalls eine ärztliche Untersuchung und setzt in $\$ 2$ Abs. $5 \mathrm{~S} .1$ zudem eine Befragung des Versicherten durch den Arzt zur aktuell ausgeübten Tätigkeit und den damit verbundenen Anforderungen und Belastungen voraus. Beides kann grundsätzlich auch in einem Fernkontakt erfolgen; beides scheint in der Hamburger Konstellation nicht hinreichend der Fall gewesen zu sein. Ebenso ist nicht ersichtlich, ob und auf welcher Basis sichergestellt wird, dass die auf dem Portal angebotene rückdatierte AU-Bescheinigung entsprechend $₫ 5$ Abs. 3 S. 3 AU-RL nur, ,ausnahmsweise und nur nach gewissenhafter Prüfung" ausgestellt wird. Auf die Frage nach der Möglichkeit von Rückdatierungen in den FAQs folgt diese Antwort: ,Sie können auswählen, ob Sie ab sofort [...] oder ab gestern [...] krankgeschrieben werden möchten." ${ }^{36}$

\section{Zumindest nicht nur am Telefon?}

Unterstützung scheint die Position des LG Hamburg in einem - in der Entscheidung aber nicht benannten Urteil des BSG aus dem Jahr 2014 zu finden. Darin hatte das Gericht in einem Revisionsverfahren $\mathrm{zu}$ einem Krankengeldanspruch die Rüge der nicht hinreichenden Sachverhaltsaufklärung zu einer telefonisch festgestellten Arbeitsunfähigkeit als nicht entscheidungserheblich zurückgewiesen: , $\$ 46 \mathrm{~S}$. $1 \mathrm{Nr}$. 2 SGB V [... setze schließlich] unabdingbar sowohl bei der Erstfeststellung der AU als auch bei nachfolgenden Feststellungen die persönliche Untersuchung des Versicherten durch einen Arzt voraus [...]. Eine telefonische Befragung [... genüge dem] nicht [...]. Dies [... folge] schon aus der durch das Wirtschaftlichkeitsgebot des $\$ 12$ Abs. 1 SGB V vorgegebenen Notwendigkeit, [... Krankengeld] nur auf der Grundlage einer bestmöglich fundierten ärztlichen Einschätzung zu gewähren. “" ${ }^{37}$ Etwas später ergänzt das Gericht dann noch das Kriterium der „Unmittelbarkeit“38 der Untersuchung. Auch bei dieser Entscheidung ist aber zu berücksichtigen, dass sie noch in einer Zeit ergangen ist, in der der Spruch, ,am Telefon und durch die Hose stellt man keine Diagnose", noch zum ärztlichen Selbstverständnis gehörte. Wenn aber selbst die Ärzteschaft zu dieser Zeit noch davon ausging, dass eine ausschließliche Fernbehandlung keine hinreichend sichere Gewähr für die erforderliche medizinische Feststellung bietet, ist es nicht verwunderlich, dass sich das BSG der Einschätzung der medizinischen Profession angeschlossen hat. $\mathrm{Ob}$ das Gericht heute unter den geänderten Vorzeichen an der Absolutheit seiner Aussage festhalten würde, ist dagegen ungewiss. Viel wichtiger ist zudem, dass sich das BSG in seinen Ausführungen ausdrücklich auf die te- lefonische Arbeitsunfähigkeitsuntersuchung bezogen und damit auch beschränkt hat. Ein Arzt-Patienten-Gespräch in einem Video-Chat, wie es bereits im Zeitpunkt der Urteilsfindung des LG Hamburg in Form der sogenannten Videosprechstunde in GOP 01439 des EBM verankert war, bietet andere Feststellungsmöglichkeiten und könnte daher gegebenenfalls auch den Anforderungen des BSG genügen.

\section{Aktuelle Entwicklung (Änderungsvereinbarung $\left.z u \int 31 B M V-\ddot{A}-C O V I D-19\right)$}

Aus Anlass der aktuellen COVID-19-Krise haben der GKV-Spitzenverband und die KÄBV am 9.3.2020 eine Änderungsvereinbarung zu $₫ 31$ des BMV-Ä getroffen. Diese dient der Entlastung der Vertragsärzte und soll zugleich der Ausbreitung des COVID-19-Virus über ärztliche Wartezimmer entgegenwirken. Inhaltlich gestattet die Vereinbarung eine vorübergehende Ausstellung von AU-Bescheinigung auch nach telefonischer Anamnese. $\mathrm{Zu}$ diesem Zweck sollen sich Ärzte durch eine eingehende telefonische Befragung persönlich vom Zustand des Versicherten überzeugen können. Die Regelung ist begrenzt auf Erkrankungen der oberen Atemwege, die keine schwere Symptomatik vorweisen. Fälle, die die Kriterien des RKI für einen Verdacht auf eine Infektion mit COVID-19 erfüllen, waren zunächst von der Regelung ausgenommen, wurden aber durch eine weitere Änderungsvereinbarung v. 23.3. 2020 nachträglich eingeschlossen. Die Attestierung der Arbeitsunfähigkeit war dabei anfangs auf einen Zeitraum von maximal 7 Tagen beschränkt, der jedoch ab dem 23.3.2020 auf 14 Tage ausgedehnt wurde. Bei dem AUTelefonat ist der Versicherte darauf hinzuweisen, dass bei einer Verschlechterung der Symptome, nach telefonischer Anmeldung in der Praxis, unverzüglich ein Arzt aufzusuchen ist. Nach Art. 2 der Änderungsvereinbarung soll es sich dabei um eine Ausnahmebestimmung handeln, die zunächst bis zum 23.6.2020 befristet ist, durch die Vertragsparteien jedoch bei Bedarf verlängert werden kann.

Durch Art. 1 einer weiteren Zusatzvereinbarung v. 11.3. 2020 wurden diese Vorgaben auf die Ausstellung von ärztlichen Bescheinigungen für den Bezug von Krankengeld bei der Erkrankung eines Kindes erstreckt. Für diese galten die engen Vorgaben von $₫ 31$ BMV-Ä jedoch bisher ohnehin nicht unmittelbar. Umgekehrt dürfte hier allerdings zu berücksichtigen sein, dass die Möglichkeit der ausschließlich fernmündlichen Krankheitsbeurteilung von Kindern durch $\$ 7$ Abs. 4 S. 3 MBO-Ä (ärztliche Vertretbarkeit) wesentlich stärker eingeschränkt sein dürfte als bei Erwachsenen. Schließlich wird häufig nur eine telefonische Befragung der Eltern möglich sein, sodass nur eine mittelbare Wahrnehmung des kranken Kindes erfolgt. Den gänzlichen Verzicht auf einen unmittelbaren ArztPatienten-Kontakt sollte die berufsrechtliche Öffnung aber gerade nicht ermöglichen. Daneben enthält die Zusatzvereinbarung Vorgaben zur telefonischen Datenübermittlung und der damit verbundenen partiellen Unanwendbarkeit der Anlage 4a zum BMV-Ä. Die Bestätigung des Versicherungsschutzes erfolgt fernmündlich durch den Versicherten selbst und die Bescheinigungen werden postalisch an diesen übermittelt. Außerdem wurde vereinbart, dass für die genannten Leistungen die GOPs 01435 und 40122 berechnungsfähig sind.

33) Ricken, in: Rolfs/Giesen/Kreikebohm/Udsching (Hrsg.), BeckOKArbeitsrecht, 54. Ed. v. 1.12.2019, \$5 EntgFG, Rdnr. 11.

34) BSG, Urt. v. 16.12.2014 - B 1 KR 25/14 -, Rdnr. 13.

35) Scholz, in: Spickhoff (Hrsg.), Medizinrecht, 3. Aufl. 2018, 25 MBO-Ä, Rdnr. 8 .

36) Vgl. https://www.au-schein.de/faq, abgerufen am 6.2.2020.

37) BSG, Urt. v. 16.12.2014 - B 1 KR 25/14 -, Rdnr. 13.

38) BSG, Urt. v. 16.12.2014 - B 1 KR 25/14 -, Rdnr. 13 
Ungeachtet der mit den Regelungen verfolgten Zielstellung der Vertragsparteien, auf eine Sondersituation durch eine vorübergehende Absenkung der Anforderungen zu reagieren, ist damit jedoch auch ein grundsätzlicher Dammbruch verbunden. Zwar lassen sich nach einer Rückkehr in den Normalbetrieb aus medizinischen und damit auch aus berufsrechtlichen Gründen sicher Teile der Lockerung zurücknehmen. Das ist in Art. 2 Abs. 2 der Änderungsvereinbarung v. 23.3.2020 auch ausdrücklich vorgesehen. $\mathrm{Zu}$ diesen dürfte etwa der sehr weitgehend erscheinende Verzicht auf die persönliche Untersuchung von Kindern gehören. Die apodiktische Behauptung, die Ausstellung von AU-Bescheinigungen verstoße in jeden Fall - und damit etwa auch bei leichten Erkrankungen der oberen Atemwege - gegen die ärztliche Sorgfalt, dürfte jedoch vom Tisch sein. Nach Beendigung der Ausnahmesituation sind die Vertragspartner des BMV-Ä daher veranlasst, die Bewährung des Systems zu evaluieren und darauf gestützte Szenarien zu beschreiben, bei denen Vertragsärzte auch künftig individuell und unter Beachtung der ärztlichen Sorgfalt entscheiden können, ob eine telefonische Befragung des Patienten zur Feststellung des Gesundheitszustands im Einzelfall genügt.

\section{Die Anwendung des, ,richtigen“ Berufsrechts}

Abschließend ist auf den bisher nur wenig diskutierten Aspekt der räumlichen Anwendbarkeit des ärztlichen $\mathrm{Be}-$ rufsrechts im Zusammenhang mit Fernbehandlungsangeboten einzugehen. Der Portalbetreiber ist in Hamburg ${ }^{39}$ angesiedelt, so dass das LG Hamburg zur berufsrechtlichen Beurteilung des Sachverhalts auf die HmbBOÄ zurückgreift. Dieses auf den ersten Blick zwingende Vorgehen kann aber durchaus Fragen aufwerfen. Zum einen ist es für den Betrieb eines Behandlungsportals nicht zwingend erforderlich, dass die dort tätigen Ärzte ebenfalls ihren Sitz im selben Kammerbezirk haben. Nach Angaben des Portalbetreibers im Internet habe dieser anfangs sogar bewusst auf in Schleswig-Holstein ansässige Ärzte zurückgegriffen, da $\$ 7$ Abs. 4 S. 3 SchlHBOÄ abweichend von $\$ 7$ Abs. 4 S. $3 \mathrm{MBO}-\ddot{A}$ und $₫ 3$ Abs. 3 S. $3 \mathrm{HmbBOÄ}$ auf das Kriterium des ,Einzelfalls“ verzichtet ${ }^{40}$. Ob dieses Modell heute weiterverfolgt wird, oder ob der Sitz der Ärzte vom Gericht überhaupt ermittelt wurde, verrät das Urteil nicht. Relevant werden könnte das etwa auch bei brandenburgischen Ärzten, denen die ausschließliche Fernbehandlung durch ihre LBO weiterhin ${ }^{41}$ untersagt ist. Diese könnten zudem bei einer - im Ergebnis abzulehnenden - Ausrichtung am Ort des Portalbetreibers problemloses forum shopping betreiben. Zum anderen stellt sich bei der Erbringung ärztlicher Leistungen über Fernkommunikationsmittel auch generell die Frage nach dem Ort der Berufsausübung. Ist dieses der Sitzort, an dem der Arzt das Portal bedient, so ist seine LBO heranzuziehen. Sowohl die Praktikabilität bei der Ermittlung als auch die mit der Kammermitgliedschaft verbundenen Sanktionsmöglichkeiten sprechen für dieses Verständnis. Andererseits erscheint es aber auch nicht als fernliegend, auf den Ort des leistungsempfangenden $\mathrm{Pa}-$ tienten abzustellen, dessen Schutz zumindest auch zu den Grundanliegen $^{42}$ des Berufsrechts gehört. Zumindest in grenzüberschreitenden Konstellationen deuten sowohl Art. 6 Abs. 1 lit. b) Rom-I-VO ${ }^{43}$ als auch Art. 5 Abs. 3 RL 2005/36/EG in diese Richtung.

\section{Fazit}

Soweit sich die Rahmenbedingungen des konkret zur Beurteilung stehenden Geschäftsmodells durch den Tatbestand der Entscheidung und die Hinweise auf der Internetseite des Anbieters aufklären lassen, ist dem LG Hamburg zumindest für diesen Einzelfall im Ergebnis zuzustimmen. Es ist davon auszugehen, dass weder die Anforderungen der S\ 7 Abs. 3 S. 3 und 25 S. 1 HmbBOÄ noch die besonderen vertragsarztrechtlichen Bedingungen für die Ausstellung von AU-Bescheinigungen eingehalten wurden. Die Begründung des Hamburger Urteils überzeugt dagegen nicht. Insbesondere stützt sie keineswegs die allgemeingültige Aussage, dass das Modell „AU-Schein nach Fernuntersuchung" nach derzeitiger Rechtslage generell ausgeschlossen sei. Uberzeugende Argumente gegen eine grundsätzliche Unzulässigkeit von Ferndiagnosen sind dem Urteil - nicht zuletzt wegen der fehlenden Auseinandersetzung mit $\$ 7$ Abs. 4 S. $3 \mathrm{MBO}-\ddot{\mathrm{A}}$ - erst recht nicht zu entnehmen.

39) Vgl. https://www.au-schein.de/impressum, abgerufen am 6.2. 2020.

40) Vgl. dazu https://tinyurl.com/qpv2ddx, abgerufen am 6.2.2020.

41) Vgl. dazu Beschl. der LÄK Brandenburg v. 8.9.2018.

42) So etwa explizit in $\$ 27$ Abs. $1 \mathrm{MBO}-\ddot{A}$.

43) Insoweit allerdings nur für das anzuwendende Behandlungsvertragsrecht. 